\title{
Can infovis tools support the analysis of spatio-temporal diffusion patterns in historic architecture?
}

\author{
Jean-Yves Blaise, Iwona Dudek \\ UMR CNRS/MCC 3495 MAP
}

\begin{abstract}
This paper's main claim is that analytical reasoning on spatio-temporal diffusion patterns requires a step into abstraction that traditional figurative solutions like maps or 3D virtual models do not encourage. Accordingly, we investigate alternative research practices, namely infovis (Information visualisation) and visual analytics, where the focus is put on revealing patterns of change, and more generally on gaining insight on individuals and collections through visual means.

We introduce four graphic combinations implemented on a test case - Zbigniew Dmochowski's architecture of Poland, a respected and comprehensive classification of architectural facts \& trends in Poland over a millennium that combines morphological, stylistic and functional division lines. The contribution presents the pluses and minuses of these combinations, the arguments behind their making, how they have shed (or not) a new light on the test case.
\end{abstract}

\section{Keywords}

Architectural heritage, Information Visualisation, spatio-temporal data, Classification.

\section{Introduction}

There are books analysts can rely on in order to analyse pieces of historic architecture in space (dictionaries, maps, etc.) or to spot the time slots they can be connected with (literature about History and History of Architecture). There are also some books that relate changes in shapes over time to a context, and thereby provide means for relations assessment: a typical example is Viollet Le Duc's encyclopaedia of architecture.

But in all three cases, graphics used almost never fully integrate the time parameter and the spatial features. They rather put them side by side, like in the classic timeline+ cartography paradigm, or provide low-level indications (a limited number of variables taken into consideration) like in the typical map+arrows or map+layers paradigms. Now maybe this was before the computer age? Well, of course not. What computer solutions can we today rely on if we need to understand and represent the patterns of diffusion of an architectural trend, in time and space? Animation techniques, where time is mapped by time? These techniques are well suited for following movements, but they also have proven inefficient in supporting notably explorative tasks.

In this contribution, we investigate how solutions stemming from the fields of Information visualisation (infovis) can reveal patterns of change, and more generally can support background tasks researchers need to carry out when analysing the evolution of historic architecture (relate findings on individual cases to general knowledge, underline contradictions, local architectural inventions, foster the understanding of the individual's position with regards to trends at that time and that place, renew the interpretation of a stylistic classification; etc).

In a state of the art preliminary section, the contribution positions the above infovis field in terms of scientific legacies, and with regards to spatio-temporal datasets. In this same section we also shortly comment on the visual solutions that will be tested and then focus on the four combinations (Fig.1) that we have implemented on a test case - Z.Dmochowski's architecture of Poland (Dmochowski, 1956). 

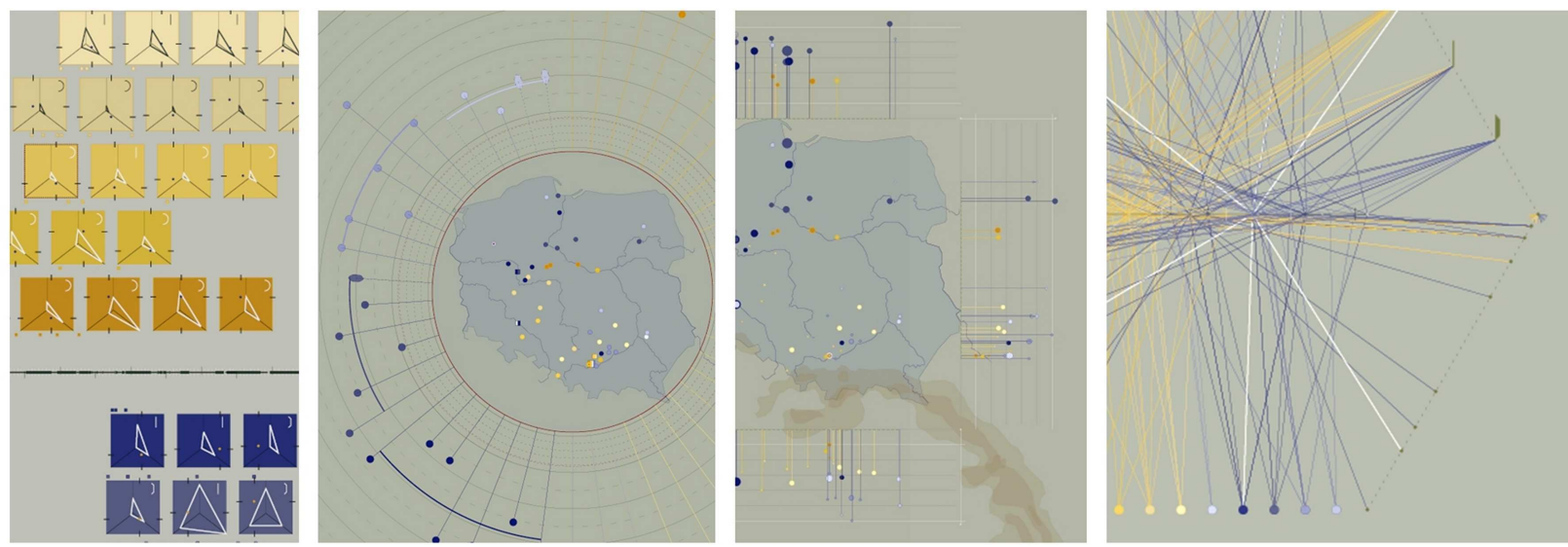

Figure 1. The four combinations, from left to right, Timeline + small multiples, concentric time, cartography + multidimensional icons, TimeWheel (Aigner at al. 2008).

However it has to be said that we shall make no general claim on the history of Polish architecture: Dmochowski's classification - although interesting by many aspects - relies on a subjective, partial selection of edifices. Our intent is to use his classification as a mean to investigate potential benefits of Infovis solutions:

- in uncovering unsaid biases,

- in uncovering spatial and temporal patterns,

- in underlining exceptions, unexpected behaviours,

- in supporting lacking / uncertain / imprecise data.

In the following sections we first come back to Bertin's vision of "graphics as an answer to a question" (Bertin 2005) and propose a short introduction to Z.Dmochowski's choices so as to underline what we wish to understand. We then present the pluses and minuses of our combinations and how they have shed (or not) a new light on the test case, before concluding on how the specificity of the data handled in historic sciences can be taken into consideration in the visual analytics process.

\section{Conceptual background}

In his clear-cut "brief history of data visualization" Michael Friendly (Friendly 2006) identifies cartography and statistics as the two main scientific legacies of infovis, quoting for instance Galton's time series or Minard's figurative maps. By choosing these examples, Michael Friendly somehow already makes the point we wish to make: at the origin of infovis is a concern for spatio-temporal data sets, a need to foster a better understanding of spatio-temporal patterns, and at the end of the day the idea that graphics can help us conduct reasoning tasks on such data sets so as to uncover unthought-of explanations. All through E.R Tufte's stunning written works further proofs are given that graphics can usefully back up analyses of trends in space and time (Tufte 2001; Tufte 1990; Tufte 1997; Tufte 2006). A number of ground-breaking contributions still emerge today on applications of infovis and related fields to spatio-temporal datasets, as exemplified in (Keim et al. 2011).

In the typical contexts of geovisualisation or GeoSpatial Visual Analytics fields, applications range for instance from analyses of moving objects (Biadgilgn et al. 2011) (Zhao et al. 2008) (Kapler and Wright 2005) - a revival of the time geography paradigm (Chardonnel 2007) - to more abstract developments like the use of visual metaphors for the assessment of semantic relations (Sabol and Scharl 2008).

In parallel, a number of contributions focus on the temporal aspects - may it be in terms of visual solution like (Havre et al. 2002)'s Theme River, or in terms of time-oriented data modelling challenges like (Aigner et al. 2008). Issues related to the production and readability of graphics - in the shadow of Bertin's graphic semiology- are also addressed such as the classic 2D vs. 3D debate (Vrotsou et al. 2010).

Yet it has to be said that infovis covers a wide range of application fields, far beyond spatio-temporal data sets - and a comprehensive overview of related works would be, if not unreachable, for sure 
irrelevant here. Moreover, the term infovis itself has here and there been complemented or replaced by other terms such as Knowledge Visualisation or Visual analytics. So we consider more fruitful to briefly introduce in the next sub-section some definitions and pinpoint some differences, before coming back to this contribution's storyline.

\subsection{Infovis \& visual analytics}

To start with a definition has to be given for the term "visualisation", a term that today can be found in literature as a sort of flabby synonym for representation, and used in the context of numerous practices (abstract diagrams, cartography, CAD and GIS, realistic rendering, etc.). As far as information and data visualisation are concerned, visualisation is not about drawing but about thinking-in the words of (Card et al 1999) it is about using vision to think. In his overview of the infovis field, R.Spence (Spence 2001) defines visualisation as a cognitive activity, underlining the idea that we rephrased in the informative modelling $15^{\text {th }}$ rule: If your graphics did not lead to new insight on your data, consider it useless (Dudek and Blaise 2006).

In other words, whereas representation is an end - the end of a cognitive process; visualisation is a mean - a mean to perform reasoning tasks all along that process.

Infovis is defined by W. Kienreich (Kienreich 2006) as the use of computer-supported, interactive, visual representations of abstract data to amplify cognition. W. Kienreich shows that an infovis solution is most often composed of three fundamental units combined inside a master visualisation: visual formalisms (using a diagrammatic visual language to convey info in an abstract way - bar charts, timelines, histograms - see Fig2.), visual metaphors (basing on real world equivalents - a city layout for a bibliography for instance), and visual models (in cases when the information is itself a real-world equivalent - typically cartography).

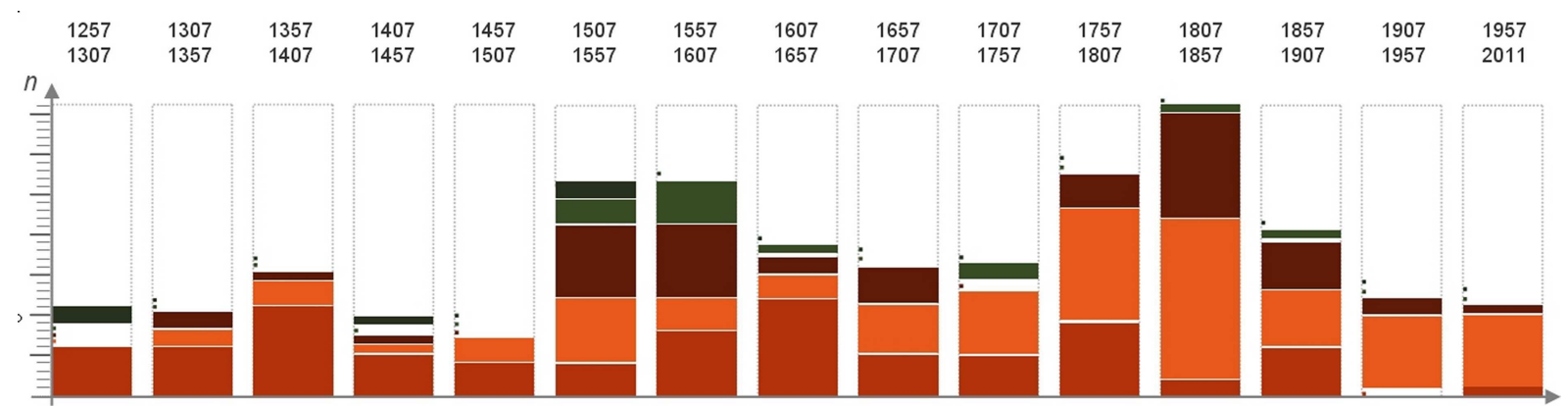

Figure 2: Represented as a classic histogram, a time series (50 years granularity) showing density of changes on Kraków's market square (Dudek and Blaise 2011).

On the $y$ axis, we represent the overall amount of changes and alternatives for the whole collection of 27 artefacts under scrutiny, counted every fifty years.

Reddish rectangles represent, from bottom to top, morphological changes (a storey added to the artefact for instances), episodic changes (basically maintenance, however can include major changes that have no impact on the artefact's morphology such as change of roof material), destructive changes (partial or total destruction).

Greenish rectangles represent confirmed (bottom) and unconfirmed (top, darker) alternatives. Confirmed alternatives mean we deal with possible divergent options on what did occur, basing on indications that are duly established and related to the artefact under scrutiny. Unconfirmed alternatives correspond to indications that may be taken into consideration or may be not because the information is questionable, or more often because it is not directly related to the artefact under scrutiny but to a neighbour, or any semantic group it may belong to (typically useful when considering possible (but unaccounted for) consequences of a fire on a neighbouring artefact)

In this visualisation note for instance for the first half of the XIXth century (1807-1857) that episodic changes (modifications - orange) widely outnumber destructive changes (brownish red). The visualisation shows the "pattern of destruction" usually associated with this period should rather be called "pattern of renunciation" in front of maintenance and repair difficulties. 
Infovis is an expanding field, with a number of branches we are not particularly interested in (in the context of this contribution). What makes an infovis solution distinguishable is the info (abstract information, large and/or complex information spaces) and the services expected (information seeking tasks, information discovery tasks).

By contrast, the sometimes disputed field of knowledge visualisation often works on smaller, but highly organized sets of information, and focuses on the transfer of knowledge among persons - may the information be abstract or not (Kienreich 2006).

Finally, the recent field of visual analytics is defined as an outgrowth of the fields of information visualization and scientific visualization: it focuses on "analytical reasoning facilitated by interactive visual interfaces" (Thomas and Cook 2005). Obviously the dispute on "which is which" is of poor relevance here. What is key to mention though is that these fields share on one hand overlapping legacies and on the other hand common techniques, goals, and sometimes methods.

\subsection{The visual solutions tested}

Over the years, a large number of visual solutions have been introduced to cope with spatio-temporal or time oriented data sets - an overview of this latter topic can be found in (Aigner et al. 2011). In this paper we shall analyse combinations of the following:

1. Timeline (Fig3, a) this is a concept we do not need to comment on very long, as its use is rather common (in particular in Western cultures where time is seen as linear). Naturally this is its major strong point: it is easily understood. In fact when analysing it from closer a timeline is strongly restrictive: it implies a de-facto discrete time model, needs continuity in the time scale to be readable, it is poor in assessing rhythms and frequencies, and furthermore it better adapted to long walls than to screens where it requires tedious zoom and pan interactions (see on this Mostern and Johnson 2008 or Blaise and Dudek 2008).

2. Small multiples (Fig3, b): this expression was introduced by E.R. Tufte (Tufte, 1990), but the concept itself is attributed by M. Friendly (Friendly, 2006) to Christopher Scheiner's 1611 solution to show the changing configuration of sunspots over time. The idea is to show in a grid-like layout successive spatial distributions of this or that object, of this or that phenomenon, thereby allowing a visual analysis of changes. This solution was for instance used by Galton in his XIXth century multivariate weather charts to uncover meteorological trends (Friendly, 2006), and applied on historical data sets in (Dudek and Blaise 2011).

3. Concentric time (Fig3, c): this is a visualisation we introduced in a recent experiment on the development of the market square in Kraków, that combines time and space. It is composed of a central 2D map, with all around it expanding concentric circles that represent a move towards the past. To each artefact positioned inside the 2D map corresponds a classic timeline, developing radially, along which events connected to the artefact's evolution can be localised in time. Various interactions between the temporal indications along the timelines and the spatial indications inside the central 2D map are then implemented to facilitate the analysis (Blaise and Dudek 2011).

4. Multidimensional icons (Fig3, d): there are a number of striking examples of how multidimensional icons can help fostering insight "at a glance" in (Spence 2001) or in (McCandless 2010). The idea is to summarise the values of several variables for a given item in a collection through a simple $2 \mathrm{D}$ icon - often relying on analogies or metaphors. In the context of spatio-temporal datasets this solution however has drawbacks. Multidimensional icons are abstract representations (spatial features are hard to convey), and the number of variables that can be compared, in a readable way, is an issue (Chan 2006). Multidimensional icons are usually extremely efficient in depicting an individual item's feature - they are less efficient in underling similarities and trends. 
5. TimeWheel (Fig3, e): this concept, first introduced in (Tominski et al, 2004), combines a classic horizontal timeline with segments distributed around the timeline in the shape of a polygon. Each segment of the polygon represents the range of values of a given attribute: for instance a length, an age, a price, etc. An item is localised in time on the central timeline, and then lines connect it to values of its attributes on each segment of the polygon. Although at first glance it may appear complex, the concept is definitely efficient in underlining trends inside a collection. In return, one to one comparisons are not that readable and naturally spatial distribution is clearly hard to convey.

6.

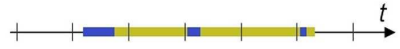

(a)

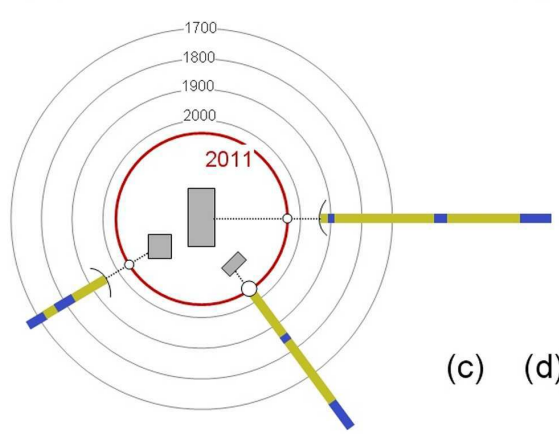

(b)

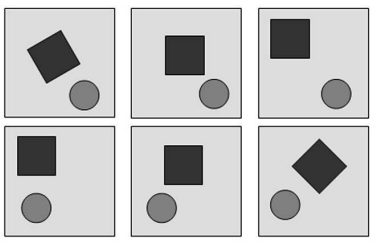

(d)

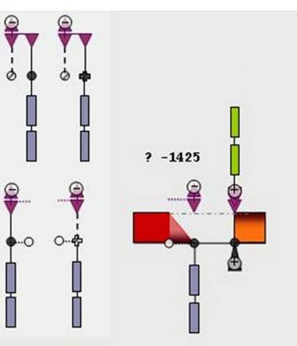

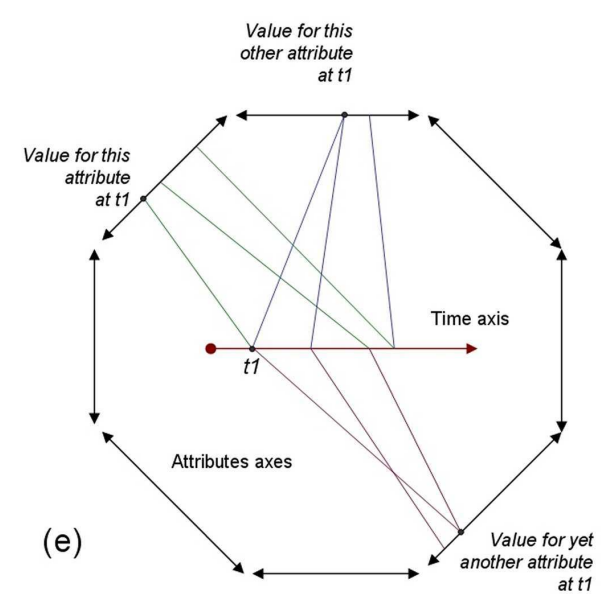

Figure 3 : Principles of the five solutions combined.

\section{The test case}

In the introduction of his historical survey, Z.Dmochowski, a Polish architect exiled in Great Britain during WWII, defines his objective as such: "show the development of architecture within the polish State from the Xth to the XIXth century" (Dmochowski, 1956, pp. xvii). Yet Dmochowski's book is more than a survey, it is a de-facto classification - yet with little indications on the division lines used. Some hints are given in the introduction: "the book divides the material according to style, but the distinctions should not be pressed too far". Dmochowski pushes this doubt further by adding "It is a great help, in studying the history of architecture of any country, to know something of its political [...] background": a way to put stylistic affiliations into a wider perspective. Finally, his definition of architecture - "[...] architecture, which of all the arts is the most socially conditioned" - further questions classic stylistic division lines. In short, Dmochowski acknowledges that styles are a key marker of trends, but in the same time that there are other aspects - context in a broad sense - that are equally key in a sensemaking classification effort.

Dmochowski's survey is basically a description and a classification of individual edifices, with varying amounts of information given depending on the edifice. For a majority, elements of chronology and a spatial layout (2D plan) are given. Other pieces of information on the morphology are more unevenly available. Edifices are segregated by groups (one group one chapter) that correspond to the third step in a three-levels classification (Fig4.):

1. At step 1, styles act as the main division line (Romanesque, Gothic, Renaissance, etc.)

2. At step 2, use and/or destination of the edifice refine the classification (Romanesque secular architecture, Romanesque ecclesiastical architecture, etc.)

3. At step 3 - groups - architectural composition and choices are introduced in order to further segregate the collection (Basilican churches with transept, Basilican churches without transept, Single cell circular churches, etc.). 

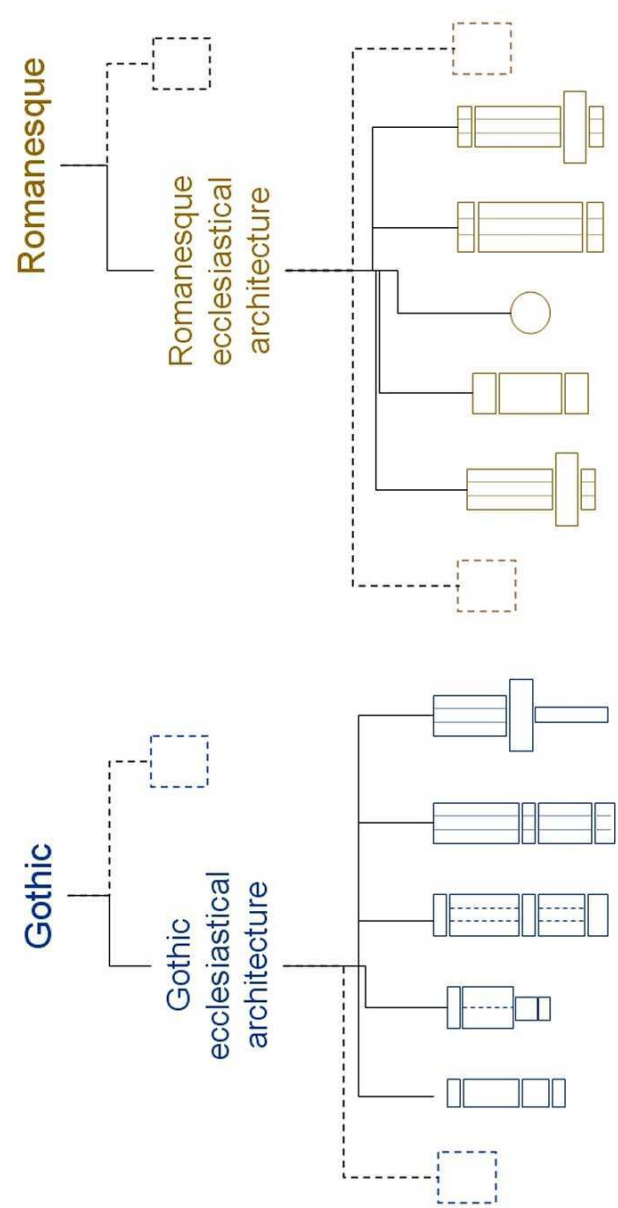

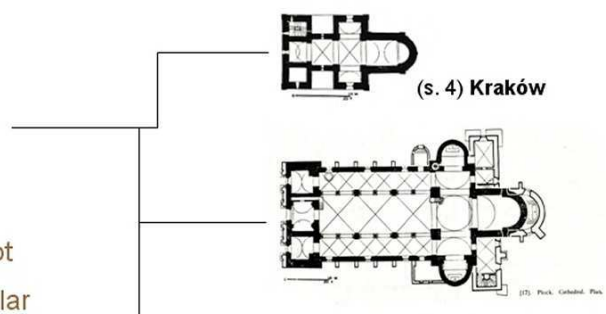

churches without transept

Group 4: single cell circular churches

Group 5: single cell rectangular cell

Group 6: Cistercian churches and monasteries
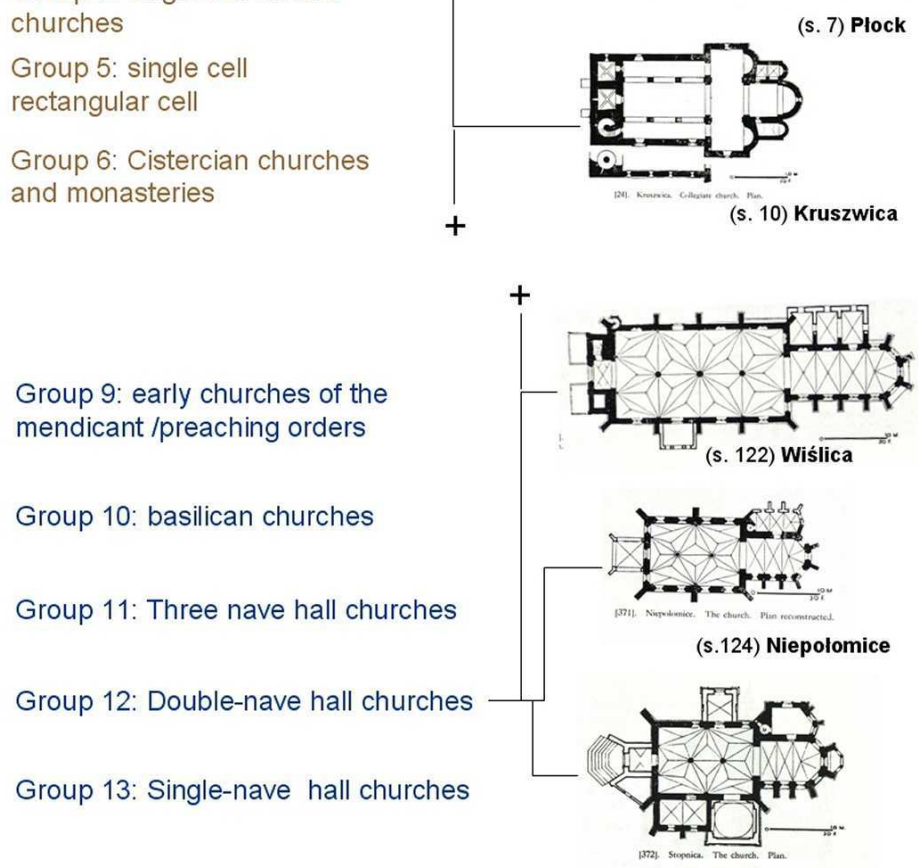

(s. 125) Stopnica

Figure 4 Classification levels, groups considered in this experiment, examples of edifices.

Beyond these three division steps there are other unsaid steps: space and time. Indications given here by Z.Dmochowski are quite hereterogeneous (dates like "XIIth century, or "1086") and furthermore this variability differs from group to group.

Now at this stage it has to be made clear that our goal was not to come out with an exhaustive analysis of Dmochowski's survey, but to conduct something like a like a proof of feasibility experiment. So the experiment we report was carried out on a subset of Dmochowski's survey: 50 ecclesiastic edifices, 2 styles (Romanesque /Gothic) 10 groups (Fig4), time and space, plus a subjective selection of features (our interpretation step : dimensional and architectural features, corresponding to heterogeneous variables, not systematically present in the survey). In short, from Dmochowski's survey we extracted an information set that can appear as rather simple, with eight variables to combine (Fig.5):

- Style

- Group

- Foundation date

- Localisation (2D coordinates)

- Length

- Shape of the Apse

- Number of chapels

- Number of Interior volumes 


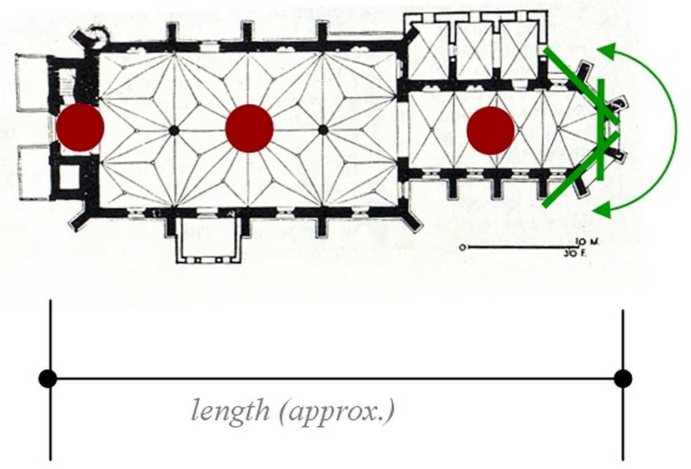

style : gothic

group : Double-nave hall churches

Foundation date : 1350

Location : Wiślica $\left(50^{\circ} 20^{\prime} 48^{\prime \prime} \mathrm{N} 20^{\circ} 40^{\prime 2} 25^{\prime \prime} \mathrm{E}\right)$

Max. length : $51,1 \mathrm{~m}$

Number of interior volumes : 3

Apse : polygonal

Number of chapels : 0

\section{Figure 5: "id card" an edifice}

Yet it might not be that simple -even with such a limited test case there are some clear difficulties:

- Unsaid division lines in Dmochowski's classification

- Heterogeneous variables

- Lacking / imprecise pieces of data

- Handling of BOTH time and space

- Need to depict each item, its relation to others, and the global features of the collection.

Well these difficulties are precisely what makes his input a great test case: our objective will be to try and see, given these difficulties, whether or not visual tools can help us uncover information and knowledge, whether they can help us gain some insight on his classification effort, on each of the 50 edifices, and on the 50 of them as a collection.

\subsection{Timeline + small multiples}

In this first combination a point corresponding to the foundation date of the edifice is localised along a classic timeline. In parallel, inspired by the small multiples precedent, we sum up inside a square the seven remaining variables through colour, value, position, shape. The square's background colour and its value are used to convey the style and group variables (Fig6, a). The square acts as an equivalent to the map of Poland - more precisely a map of Poland is inscribed inside the square. As a consequence the localisation (2D geographic coordinates) of the edifice can be interpreted as $(\mathrm{x}, \mathrm{y})$ coordinates inside the square (Fig6, b). Values for length, number of chapels and number of interior volumes are reported on three axes, forming a triangle (Fig6, c). Finally, a small icon on the top right corner identifies the shape of the apse (Fig6, d).

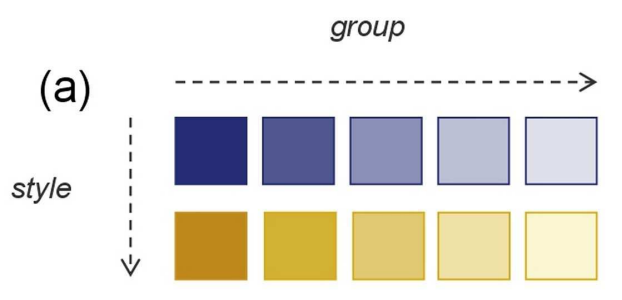

(c)<smiles>CC(C)C</smiles>

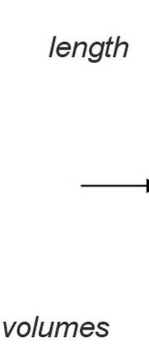

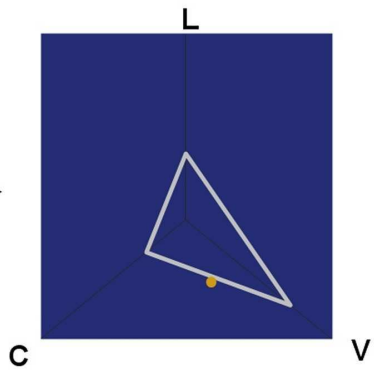

(d)

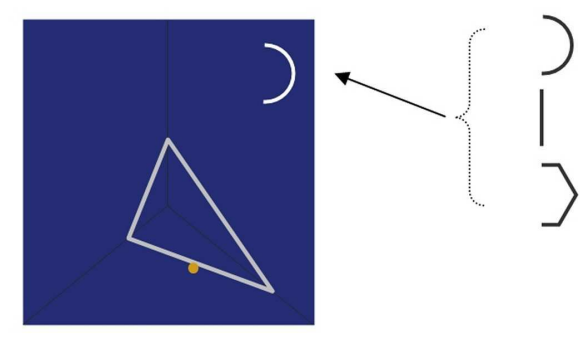

Figure 6: visual encoding of an edifice's eight variables. 
But then how can these squares be connected to a chronology, to the timeline? If we just align them with the timeline there will be either clumsy overlapping when edifices are close in time - or a lengthy visualisation unsuited to a context view (the whole collection within the eyespan). In fact this combination is not well suited to reading the chronology in discrete or continuous time. Instead, we use it to read the chronology in ordinal time (only the order is known): each group is represented with its oldest item duly aligned on the timeline (Fig7, a), and the rest of the group simply positioned horizontally by order of appearance. Interactions on each small multiple do allow the reader to precisely date it on the timeline (Fig7, c).

The result is a clear insight gained on the variability of items inside each group, and accordingly on groups consistencies (Fig7, b). Exceptional behaviours for one specific variable are also underlined (Fig7, d), as well as cases when the dating is questioned or imprecise (Fig7, e). A point can for instance be made on this subset of Dmochowski's survey: the most "recent" group the least accurate in terms of dating...

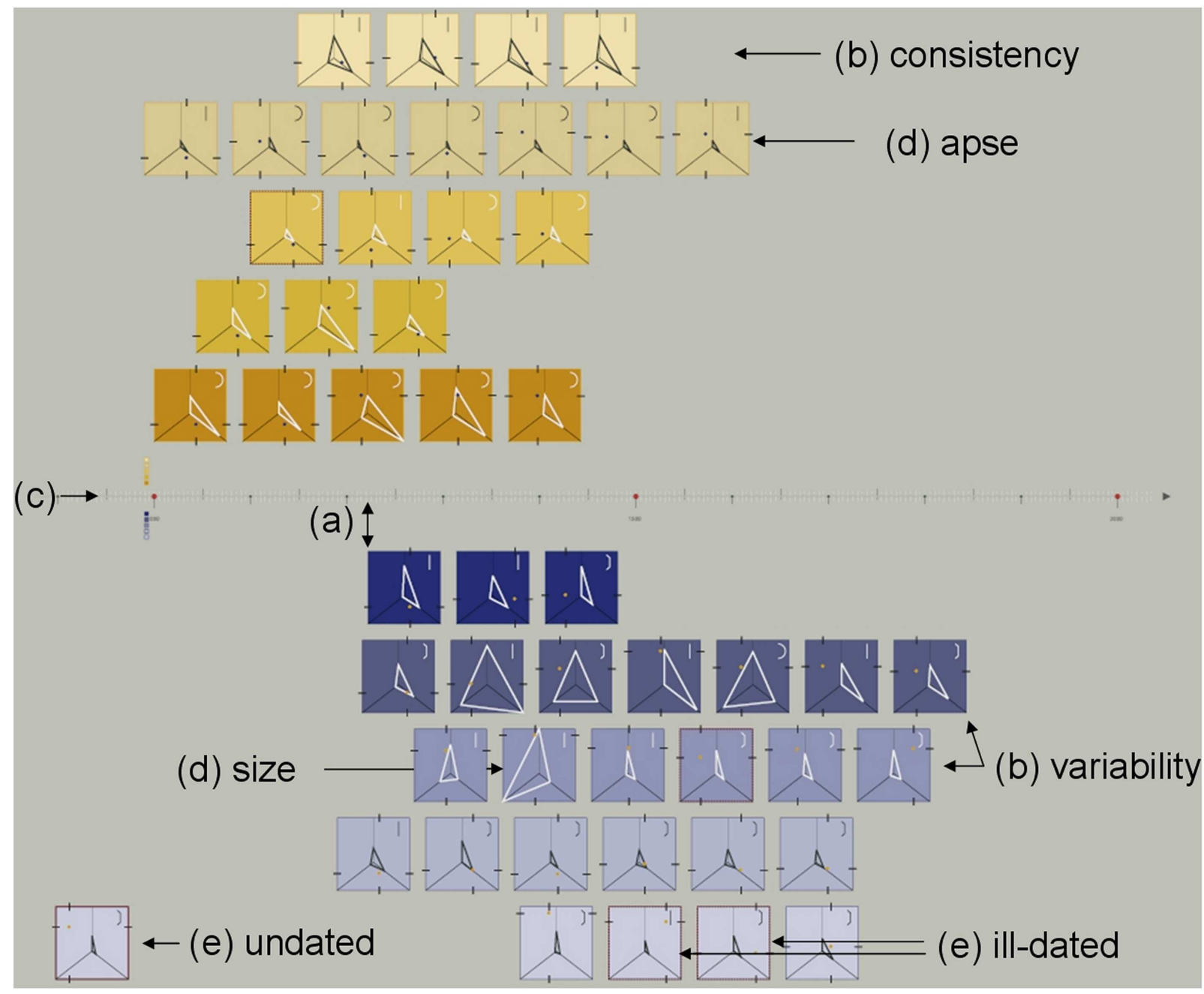

Figure 7: Combining multiples in ordered time .

A projection of each square along the timeline at a position corresponding to the actual foundation dates for each edifice can be added so as to highlight densities of change, or to correlate changes to other factors.

This combination does have its weaknesses: ordinal time is convenient when wanting to reveal group behaviours, but costly in terms of readability for individual items. Furthermore, as it is, from this visualisation only a rather vague understanding of the consistency of a group in space is within reach.

\subsection{Concentric time + small multiples}

This combination reuses the small multiple introduced above, but with the ambition to better handle 
and visualise the space parameter. A map of Poland is drawn in the central spatial layout of the visualisation, with coloured dots that position edifices (colour and value are used to convey the style and group variables). In cases when several edifices are localised in the same city rectangles are used instead of a dot. To each edifice also corresponds a dot on the concentric circles - the older the farther from the central spatial layout. Each item in the collection, each edifice, is thereby localised in time and positioned in space. On user demand items can be browsed one by one, opening the small multiple and a plan view. This modality corresponds to a focus view: it allows one to one feature comparisons inside a group, between neighbouring edifices, etc. Alternatively the whole collection of small multiples can be shown together. This modality corresponds to a context view: it delivers an overview of spatial and temporal features across the collection.

At this stage the visualisation does allow a better analysis of spatial features, but groups are not as readable. In addition, by scattering squares all around the visual display, it in fact has little to do anymore with the very concept of small multiples. This combination can however be noticeably ameliorated when representing a group by an arc located at a position corresponding to the average foundation date for edifices of the group.

When doing so, temporal consistency inside the group is clearly highlighted (Fig8, a) - a point that could not be made with the previous solution. Furthermore, if interaction is implemented between the arc representing the group and the dots on the map, clear (yet unsaid in Dmochowski's survey) spatial patterns are revealed. For instance, group 9 "Double nave hall churches" appears typical of Małopolska (south-east, around the former Polish capital Kraków) whereas group 8 "Three nave hall churches" is widespread rather in the northern half of Poland (Fig8, b).
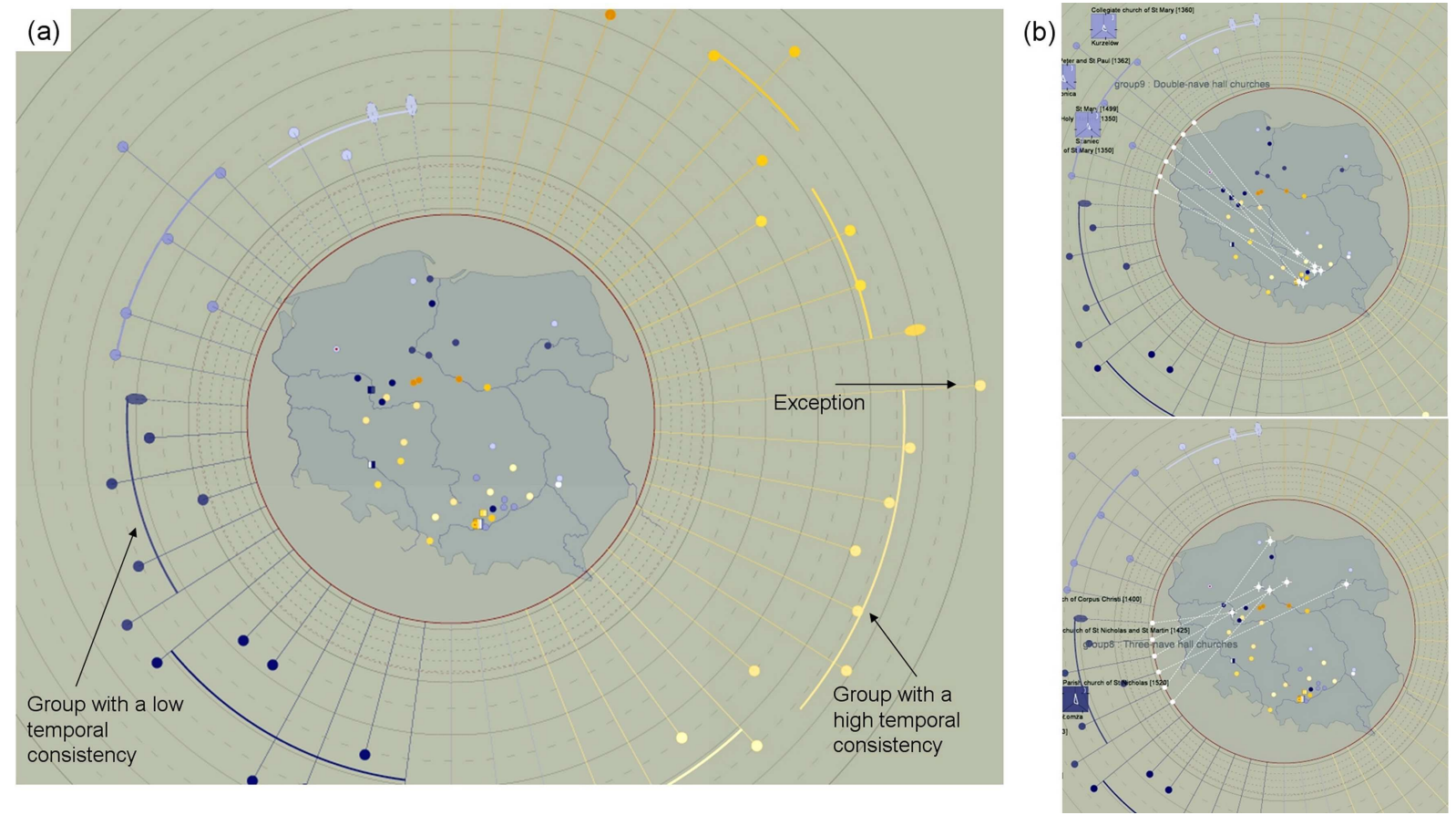

Figure 8: Assessing group behaviours with the concentric time visualisation .

In short, this solution probably has a higher potential in rendering both spatial and temporal aspects. Yet it implies a steeper learning curve, and needs stronger interactions.

\subsection{Cartography + multidimensional icons}

When handling geo-related pieces of data one of the first reaction analysts tend to have is to produce some kind of cartography. And there is no reason to believe cartography can't be used in the context of infovis solutions. In this third combination we start from a basic 2D cartography of Poland on which we position edifices as we did in the previous solution (coloured dots). Each edifice is identified by the system as belonging either to the north or to the south part of Poland, and then either to the east or to west part of Poland. As a result each edifice on the map can be connected to two multidimensional 
icons located on the sides of the map (Fig9, a). The remaining variables are summarised in a multidimensional "flower-like" icon composed as follows:

- Length of the stalk corresponds to the distance, in time, of the current edifice to the oldest inside the collection (here 990). The longer the stalk, the younger the edifice (Fig9, b).

- Diameter of the corolla's centre: length of the edifice (Fig9, c).

- Number of petals: number of interior volumes (Fig9, d).

- Shape of bottom leaf : shape of the apse(Fig9, e).

- Number of leaves: number of chapels (displayed on user demand).

Ill-defined positions and dating are represented by line styles and colour.

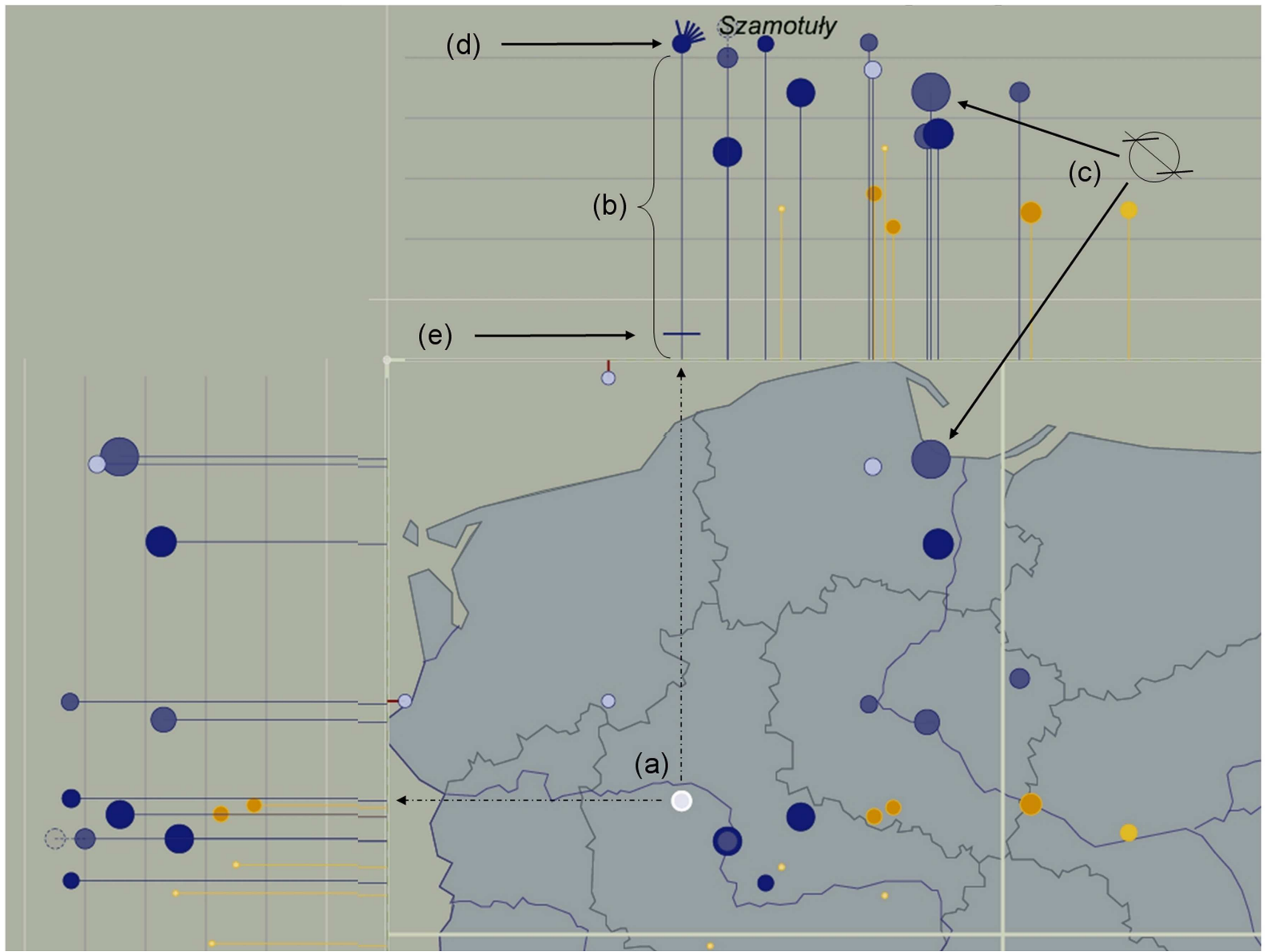

Figure 9: Combining a basic cartography with multidimensional icons .

Used in context view (Fig 10, a), the visualisation allows east/west; north/south comparisons. It helps for instance uncovering different distribution patterns between the west (regular distribution in space) and the east (imbalanced distribution in space), or between the south (high diversity in groups present, consistent and long lasting time distribution) and the north (less diversity in groups, smaller temporal coverage).

Used in focus view (Fig 10, b), the visualisation allows item to item comparisons among neighbours and helps for instance reading spatially consistent group behaviours.

On the overall, this solution over-performs the previous ones on one aspect mainly: its capacity to superimpose some layer of spatial analysis - exemplified here by the four sectors tested.

However the solution also has its drawbacks -the spatial analysis layer requires a high level of interaction, and temporal aspects are seemingly rendered with less efficiency. In detail, some choices on the making of the multidimensional icon are questionable - typically representing a $1 \mathrm{D}$ value by a 2D object (mapping the length to the diameter of a circle) is a notably risky strategy. 

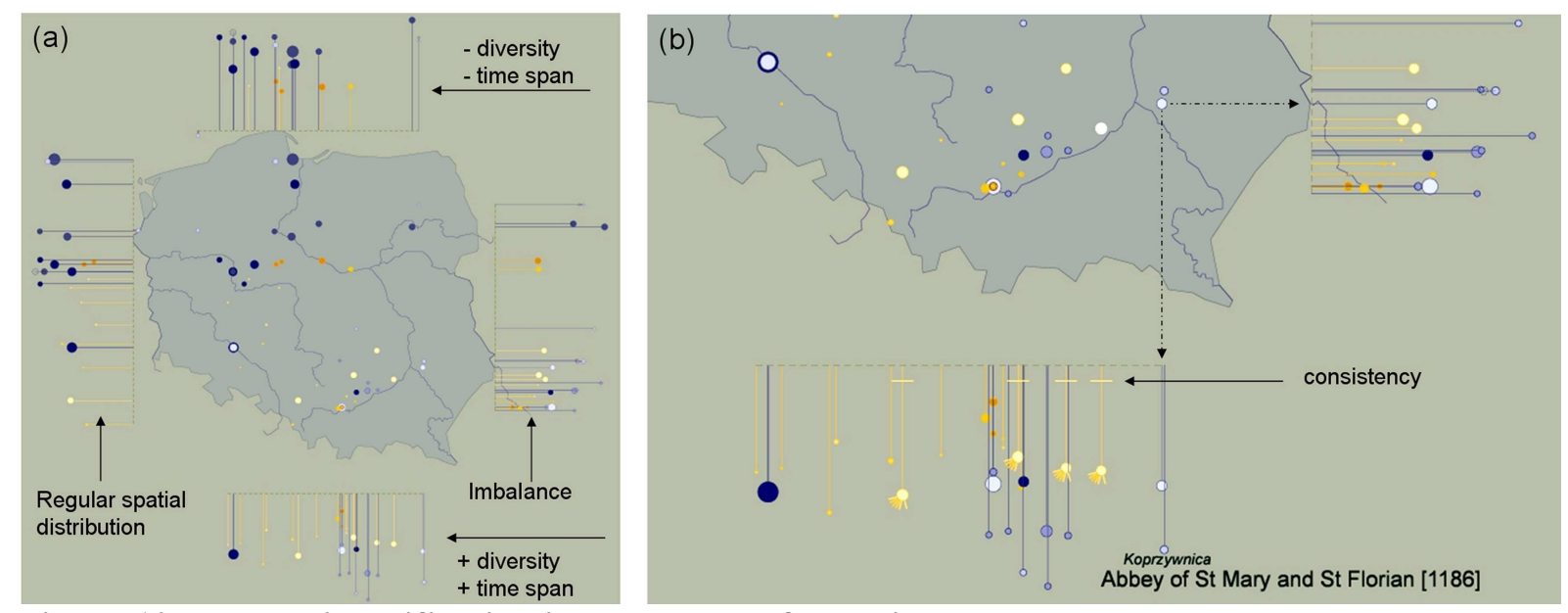

Figure 10: Pattern identification in context and focus views.

Before developing the fourth solution it is important to make two intermediary statements.

- Whereas with cartography one tends to pack everything up inside a unique system of representation - in this approach - infovis applied to historic data sets - we rather make use various systems of representation.

- Up to here the three solutions proposed remain item-oriented: each edifice is represented separately, here by a small multiple, there by a multidimensional icon, etc. Accordingly these solutions allow one-to-one comparisons, but are not necessarily well suited to analyses of the collection as a whole. Typically, if my question is "are apses in majority polygonal" the above graphics can give the answer, but with a boresome decoding effort from the user.

\subsection{TimeWheel}

The TimeWheel concept (Aigner et al, 2008) combines a horizontal timeline with segments distributed it, along which values of various attributes can be reported. In our experiment foundation dates are distributed along the central timeline, the length of which being this of the time interval between the "oldest" and the "youngest" edifice in our collection.

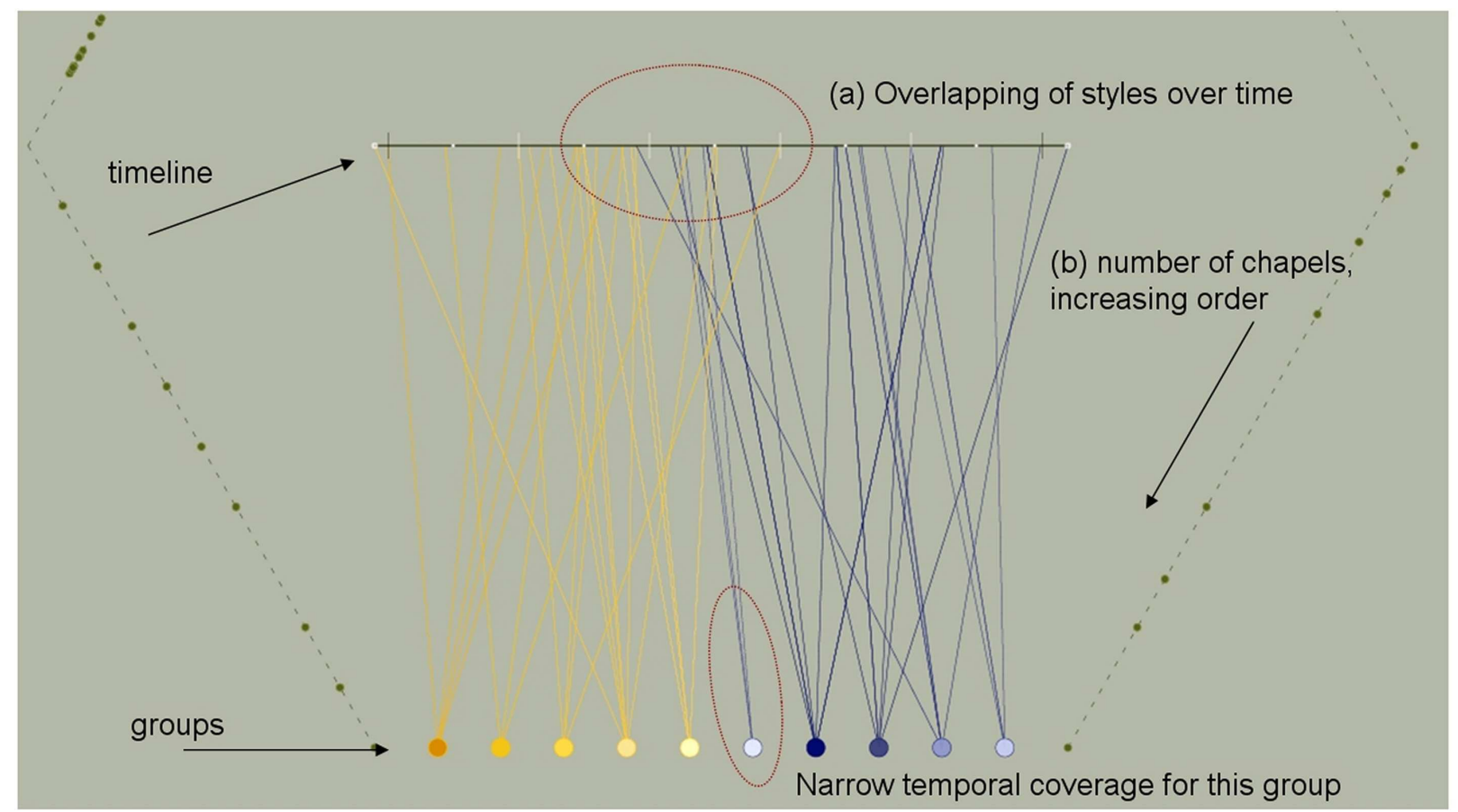

Figure 11: The TimeWheel concept, all variables values hidden except for groups.

The polygon used is a hexagon, with a bottom side used to convey two variables: groups and style, 
reusing colour and value as for the previous combination (Fig12, a). On either side of the hexagon are four segments used to convey the variables length, shape of the apse, number of chapels, and number of interior volumes (Fig12, b). Finally, we chose here to substitute to the geographical coordinates of the edifice another indication: the size (number of inhabitants) of its host city today (Fig12, c). At first glance the visualisation may appear rather visually crowded - but with some interaction it reveals its full potential (the time overlapping of styles is for instance clear-cut to read Fig11, a).

We represent on each segment all the values for the corresponding variable inside the collection by a point (for instance, all lengths are aligned in increasing order). As a result densities are straightforward to read (Fig 11, b).

Probably more interesting is the ease with which this graphics helps spotting expected patterns and exceptional behaviours. In the case we illustrate hereafter the visualisation shows that only one edifice classified by Dmochowski as "gothic" has a round apse (Fig12, d). When interactively highlighting this particular edifice (Fig12, e) further indications are given that can help the analyst get an overall idea of where this particular edifice stands with regards to other features (exceptional high amount of chapels in Fig12, f for instance).

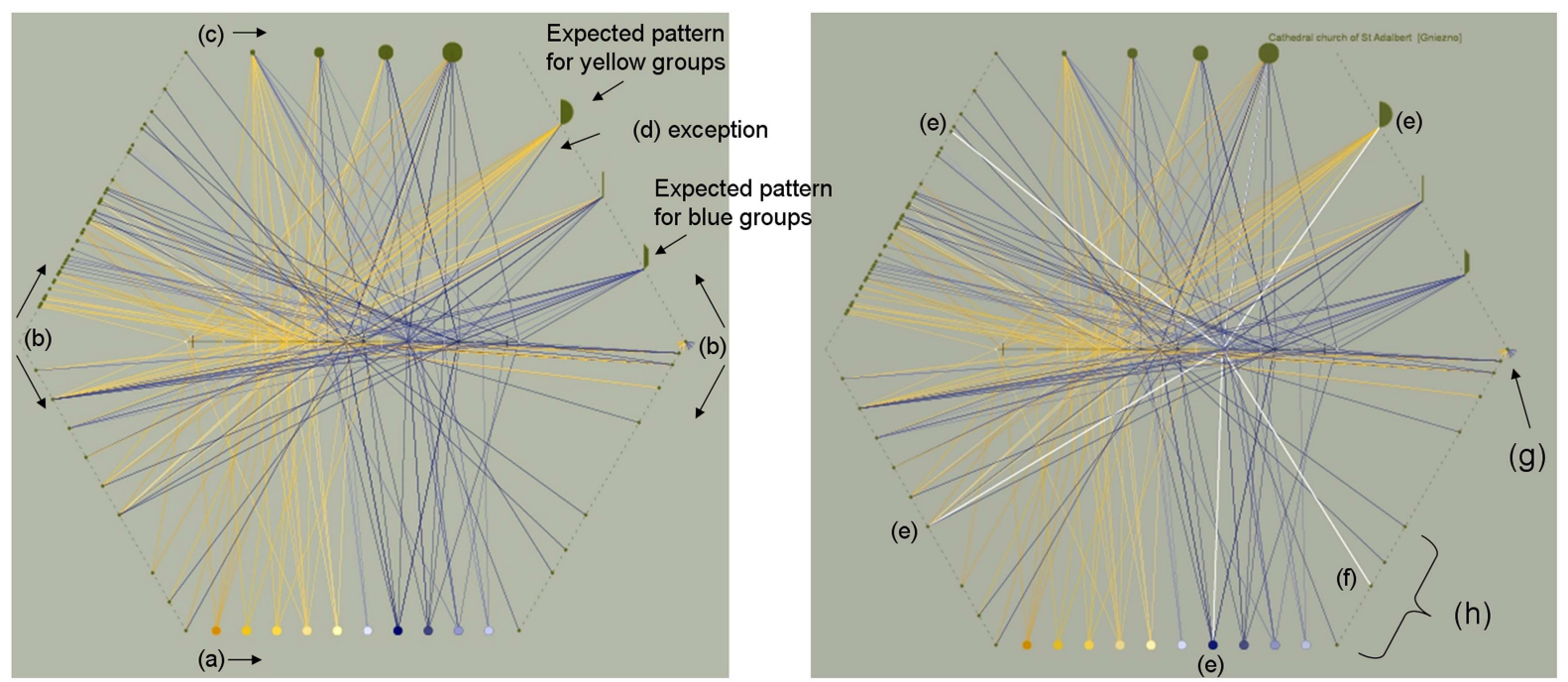

Figure 12: Distribution and cross examination of the variables.

Finally, this visualisation proves efficient in analysing how the value of a given variable varies over the whole collection. In the example above (number of chapels) value "zero" - no chapels - occurs almost in every group and style (Fig 12, g). By contrast, high values always occur inside the same 2 groups (Fig 12, h).

However, the "spatial distribution" feature is noticeably absent from this visualisation. This calls for a general remark: when dealing with spatio-temporal data sets in the context of history, one probably needs to come out with a specific, fine-tuned blend between abstraction - the infovis legacy - and figuration - the storytelling legacy.

\subsection{Interaction and implementation}

In the context of this written contribution we basically show screenshots of a set of computersupported, interactive graphics. These graphics are created dynamically, at query time, from the reading of an RDBMS system storing the actual data. They are written in the standard SVG (Scalable Vector Graphics) format (ASCII format). The whole process is supported by ad-hoc programming, avoiding dependence on any piece of commercial software. Accordingly the can be easily duplicated, provided some knowledge of RDBMS and basic programming skills are at hand.

Graphics can be read on standard web browsers, and freely distributed. Interactions can be attached to each geometric object inside the SVG file, may it be a simple background line or a group - a multidimensional icon for instance.

We do acknowledge that a number of issues are left uncommented in this paper (possible data preprocessing here and there, escaping from two-dimensional visualisations, cognitive load and 
evaluation in general, etc.). The fact is that they would drive us too far from the paper's main claim, and require quite extensive (and unplanned by the editors) explanations since visualisations we tried out would each call for specific comments.

\section{Conclusion}

The first conclusion we draw can be seen as a tribute to Bertin's view: since efficient graphics are those designed as answers to a question, it is a necessity when facing several questions, to promote several graphics. Indeed, the four solutions we have tested shed different lights on our data set. Accordingly there is nothing like a universal visualisation - there are only visualisations that do help uncovering information, and the others - useless visualisations.

Coming back to the test case, the visual solutions we have proposed did help us point out some interesting figures from Dmochowski's survey:

- basic spatio-temporal patterns, like a move northbound as time and styles pass by,

- architectural patterns, like higher or lower consistency in the architectural composition features depending on the groups,

- unsaid biases, like a relative (and questionable) homogeneity in the number of items presented in each group.

But it would take more than a sub-set of the survey, more detailed architectural analysis of the edifices chosen, more cross-examination with historic factors, to really question Dmochowski's classification.

At first glance, what we have proposed in this contribution is a method to re-read, re-interpret existing spatio-temporal data sets. From closer, we have exemplified this approach through a set of graphics that each with its pluses and minuses help uncovering patterns of the underlying data set, and have shed a new light on Dmochowki's classification effort.

Infovis and its outgrowth visual analytics are precisely about that: re-investigating a data set through visual means, and thereby giving ourselves chances to reach to some new conclusion, or at least to renew the way we picture the data set to ourselves.

However methods, tools and practises from these fields rely on human reasoning capabilities: the graphics by themselves are of no use if

1. They are not the result of adequate modelling and filtering choices (for instance keeping tracks of doubts),

2. They are dedicated to communication rather than to perform reasoning tasks

We hope this contribution showed infovis / visual analytics solutions can apply to test cases like ours, and can be fruitful even when dealing with history-oriented data sets. In the same time it has to be stressed not all of the infovis / visual analytics methodology - solutions from visual analytics are not solutions unless we fully master the process from data modelling to the production and coding of graphics. In general scientists dealing with history-related data sets should be cautious with statisticsoriented formalisms and/or automatic methods, like for instance clustering (possibly deceptive).

To conclude, we wish to add that infovis solutions should not be seen as not yet another technological magic wand - but they can, and probably should, be seen as yet another mean for us to re-examine data sets in the light of our knowledge of historical data sets.

\section{References}

Aigner, W., Miksch, S., Schumann, H., Tominski, C. 2011. Visualization of Time-Oriented Data Human-Computer Interaction Series: Springer.

Aigner, W., Miksch, S., Müller, W., Schumann, H., Tominski, C, 2008. "Visual methods for analyzing time-oriented data". Transactions on visualization and computer graphics vol 14 pp 47-60.

Biadgilgn, DM, Blok, CA, Huisman, O. 2011 "Assessing the Cartographic Visualization of Moving Objects" Momona Ethiopian Journal of Science Vol 3, No 1.

Bertin, J. 2005 (or. ed 1967). Sémiologie graphique Les diagrammes - Les réseaux - Les cartes (Semiology of Graphics Diagrams, Networks, Maps) Paris: EHESS. 
Blaise, J.Y., and Dudek, I., 2011 "Concentric Time: Enabling Context+Focus Visual Analysis of Architectural changes" In Foundations of Intelligent Systems, edited by M. Kryszkiewicz, H. Rybinski, A. Skowron, W. Raś, 632-641, Lecture Notes in Computer Science, Berlin, Heidelberg : Springer-Verlag.

Blaise, J.Y., and Dudek, I. 2008 "Experimenting timelines for artefacts analysis: from time distribution to information visualization" In Proceedings VSMM 2008 Digital Heritage, Archeolingua, Budapest : 197-204

Card, S.K., Mackinlay, J.D and Schneiderman, B. 1999 Information vizualisation: Using vision to think. San Francisco :Morgan-Kaufmann.

Chan W.W-Y., 2006 “A Survey on Multivariate Data Visualization” Technical Report, HKUST (Hong Kong University of Science and Technology),

http://www.cse.ust.hk/ wallacem/winchan/research/multivis-report-winnie.pdf, Accessed June 20, 2012.

Chardonnel, S., 2007 "Time-geography: individuals in time and space" In Models in Spatial Analysis, edited by Lena Sanders, 97-122, London: ISTE

Dmochowski Z., 1956. The architecture of Poland - an historical survey. The Polish research centre, London.

Dudek, I., and Blaise, J.Y., 2006. "informative modelling” MiaJournal 1

http://www.map.archi.fr/mia/journal/ Accessed June 22, 2012

Dudek, I., and Blaise, J.Y. 2011 "Visualizing alternative scenarios of evolution in heritage architecture" In Proceedings of the 11th International Conference on Knowledge Management and Knowledge Technologies, ICPS: ACM International Conference Proceeding Series New York :ACM.

Friendly, M. 2006. "A brief history of data visualization". In handbook of computational statistics: data visualization edited by C.Chen W.Hardle A.Unwin, 15-56. Heidelberg: Springer-Verlag.

Havre, S., Hetzler, E., Whitney, P. and Nowell, L. 2002. "ThemeRiver:Visualizing Thematic Changes in Large Document Collections", IEEE Transactions on Visualization and Computer Graphics, 8(1): 9 20.

Kapler, K., Wright, W., 2005 "GeoTime Information Visualization" Information Visualization archive 4 (2) : 136 - 146 Palgrave Macmillan.

Keim, Daniel., Kohlhammer, Jörn., Ellis, Geoffrey., Mansmann, Florian,. 2011. Mastering The Information Age - Solving Problems with Visual Analytics. Konstanz : University of Konstanz http://www.vismaster.eu/

Kienreich, W., 2006. "Information and Knowledge Visualisation - An Oblique View" MiaJournal 0(1): 7-17 http://www.map.archi.fr/mia/journal/ Accessed June 22, 2012

McCandless, D., 2010. Information is beautiful. London: HarperCollins.

Mostern, R. and Johnson, I. 2008. "From named place to naming event: creating gazetteers for history”, International Journal of Geographical Information Science, 22:10, 1091 - 1108

Sabol, V., Scharl, A.: 2008 "Visualizing Temporal-Semantic Relations in Dynamic Information Landscapes". In Proceedings of the 11th International Conference on Geographic Information Science AGILE-2008, Girona Agile council 1-6 http://geoanalytics.net/GeoVis08/a15.pdf

Spence,R., 2001. Information Visualisation. Boston:Addison-Wesley/ACMPress.

Thomas, J.J and Cook, K.A. 2005 Illuminating the Path: The Research and Development Agenda for Visual Analytics IEEE CS Press.

Tominski, C., Abello, J., and Schumann H., 2004 “Axes-Based Visualizations with Radial Layouts," in Proceedings ACM Symposium on Applied Computing 1242-1247 ACM Press. 
Tufte, E.R., 2001 (or. ed. 1983) The Visual Display of Quantitative Information. Cheshire, CT: Graphics Press.

Tufte, E.R., 1990 Envisioning Information. Cheshire, CT: Graphics Press.

Tufte, E.R., 1997 Visual Explanations: Images and Quantities, Evidence and Narrative. Cheshire, CT: Graphics Press.

Tufte, E.R., 2006 Beautiful Evidence. Cheshire, CT: Graphics Press.

Vrotsou, K., Forsell, C., Cooper, M., 2010 "2D and 3D representations for feature recognition in time geographical diary data" Information Visualization 9(12/2010): 263-276

Zhao, J., Forer, P., Harvey, A.S, 2008. "Activities, ringmaps and geovisualization of large human movement fields". Information visualization 7 (3-4): 198-209 Nature Publishing Group / Palgrave. 